\title{
Falta de articulación entre la educación media y la universitaria y sus consecuencias en los primeros años de la educación superior en zonas periféricas de Pilar. Periodos 2019-2020
}

\author{
Hilaria Armoa Quintana \\ armoahila@gmail.com \\ Universidad Nacional de Pilar \\ Facultad de Humanidades y Ciencias de la Educación
}

\section{RESUMEN}

La investigación estudia la falta de articulación entre la educación media y la universitaria y sus consecuencias en los primeros años de la educación superior en zonas periféricas de Pilar. Periodos 2019-2020. Se realizó una investigación de tipo cuali-cuantitativo de nivel descriptivo. La investigación aborda una problemática emergente no solo del país sino también de varios países Latinoamericanos, conforme a las lecturas de otras investigaciones; por lo que merece ser estudiado la manera que está afectando la realidad educativa de los estudiantes, la articulación del Nivel Medio al Nivel Universitario. Se ha llegado a la concusión que la falta de articulación entre el Nivel Medio y la universitaria es una realidad que se vive en las aulas, y que sin embargo no se la dimensiona en su real magnitud, pues de lo contrario dejaría de ser considerado un hecho que habitualmente es tratado con medidas improvisadas, casuales, que no pasan de ser meros comentarios entre directivos y docentes a un tema serio de interés, objeto de propuestas bien planificadas, coordinadas y ejecutadas desde la administración central pero con la participación real y comprometida de los principales responsables del hecho educativo. La falta de articulación en los primeros años de la educación universitaria y la educación media es una realidad evidenciada sobre todo para una población con menos posibilidades educativas como son las zonas periféricas.

Palabras clave: Falta de articulación; Educación media; Educación universitaria; Zonas periféricas. 


\title{
Lack of articulation between middle and university education and its consequences in the early years of higher education in peripheral zones of Pilar. Periods 2019-2020.
}

\begin{abstract}
The research studies the lack of articulation between secondary and university education and its consequences in the first years of higher education in peripheral areas of Pilar. Periods 20192020. A descriptive-level quali-quantitative research was carried out. The research addresses an emerging problem not only in the country but also in several Latin American countries, according to the readings of other research; For what deserves to be studied the way that the educational reality of the students is affecting, the articulation of the Middle Level to the University Level. It has been concluded that the lack of articulation between the Secondary Level and the university level is a reality that is lived in the classrooms, and that nevertheless it is not dimensioned in its real magnitude, because otherwise it would cease to be considered a a fact that is usually treated with improvised, casual measures, which do not go from being mere comments between managers and teachers to a serious topic of interest, the object of wellplanned, coordinated and executed proposals from the central administration but with the real and committed participation of those mainly responsible for the educational event. The lack of articulation in the first years of university education and secondary education is a reality evidenced above all for a population with fewer educational possibilities such as peripheral areas.
\end{abstract}

Keywords: Lack of articulation; Middle education; University education; Peripheral areas.

Artículo recibido: 03 nov. 2020 Aceptado para publicación: 07 dic. 2020

Correspondencia armoahila@gmail.com Conflictos de Interés: Ninguna que declarar 


\section{INTRODUCCIÓN}

El estudio hace referencia a las principales consecuencias evidenciadas en los alumnos de los primeros años de las diferentes Carreras de la Universidad Nacional de Pilar y la Educación Media de las instituciones educativas de zona periférica de la Ciudad de Pilar, por la falta de articulación entre la Educación Media y la Universitaria.

En tal sentido, el estudio analiza las dificultades de aprendizaje que manifiestan los estudiantes, los problemas de adaptación al nuevo ambiente y las características como se desarrollan las relaciones de convivencias entre los mismos y las características sociales de los alumnos.

Entendiendo que la adecuada articulación entre los distintos niveles de enseñanza y, en particular entre la Educación Media y la Universidad, es un requisito que marcara el éxito del estudiante en su vida universitaria, por lo tanto, ante el conocimiento de la exigencia de un equilibrio en un nivel medio a un nivel universitario se presenta esta investigación considerada un tema que merece especial atención.

Las experiencias de intentos articuladores ensayados en algunos países latinoamericanos no han logrado trascender y convertirse en modelos de políticas y/o de acción, sino más bien se han quedado en buenas intenciones por los muchos problemas que han presentado en su conceptualización y operacionalización.

Al respecto (Restrepo y García, 2017), considera que en la actualidad se cuestiona la calidad de la educación como fenómeno social, que brinde a los sujetos que se encuentran en formación la posibilidad de potenciar competencias y habilidades para insertarse en la educación superior y en el mercado productivo del país.

En este sentido, los programas técnicos y de profundización de la educación media juegan un papel imperante en la medida en que posibilitan que los estudiantes desarrollen competencias y habilidades necesarias para incursionarse a la educación superior.

La problemática de la articulación entre la Educación Media es compleja en el país, porque afecta a dos niveles de la educación, con sus propias reglas, administración, currículo y metodologías.

\section{OBJETIVOS DE LA INVESTIGACIÓN:}

\section{General}

Determinar las principales consecuencias evidenciadas en los alumnos de los primeros años de las diferentes Carreras de la Universidad Nacional de Pilar y la Educación Media de las instituciones educativas de las zonas periféricas de la Ciudad de Pilar, por la falta de articulación entre la Educación Media y la Universitaria, periodo 2019-2020. 


\section{Específicos}

1. Describir las características socio demográficas de los alumnos procedentes de las zonas periféricas de la Ciudad de Pilar y su relación con su rendimiento académico.

2. Identificar los obstáculos que enfrentan los estudiantes durante los procesos de interacción sistémica, entre todos los actores educativos, orientados a establecer las relaciones funcionales y significativas entre sus componentes.

3. Señalar las dificultades de adaptación al nuevo ambiente que presentan los estudiantes investigados, como resultado de su paso de la Educación Media a la Universitaria.

\section{JUSTIFICACIÓN}

La investigación aborda una problemática emergente no solo del país sino también de varios países Latinoamericanos, conforme a las lecturas de otras investigaciones; por lo que merece ser estudiado la manera que está afectando la realidad educativa de los estudiantes, la articulación del Nivel Medio al Nivel Universitario.

No se conoce un trabajo de investigación similar a la presente por lo que se considera necesaria e importante ahondar dicho tema.

Se cuenta con los recursos humanos quienes son los estuantes de los primeros años de las diferentes carreras de la Universidad Nacional de Pilar y que son varios los de origen de zonas periféricas de la Ciudad de Pilar.

\section{METODOLOGÍA}

La investigación se enmarca en un estudio de tipo descriptivo correlacional porque trata de demostrar la relación que existe entre la falta de articulación y el fracaso académico por parte de los estudiantes universitarios en sus primeros años de la carrera Universitaria.

Aborda los métodos cuali cuantitativo. El Método mixto es definido por Chen (2006) citado por Sampieri (2014) como la integración sistemática de los métodos cuantitativo y cualitativo en un solo estudio con el fin de obtener una representación más completa del fenómeno (p.334). Se utilizaron fuentes primarias; libros, artículos científicos y revistas. Al respecto Sampieri y otros (2014) señalan que las fuentes primarias proporcionan datos de primera mano, ya que se trata de documentos que incluyen extensas revisiones e investigaciones sobre el tema elegido (p.60).

Como fuentes secundarias: se recurrió a datos de Archivo institucional (planillas de calificaciones, de asistencia entre otros)

De acuerdo con Hernández, Fernández y Baptista (1998), ’la validez en términos generales, se refiere al grado en que un instrumento realmente mide la variable que pretende medir" (p.243). 
Tamayo y Tamayo (1998) considera que validar es "determinar cualitativa y/o cuantitativamente un dato" (p. 224). Esta investigación requirió de un tratamiento científico con el fin de obtener un resultado que pudiera ser apreciado por la comunidad científica como tal. Las técnicas de obtención de datos fueron: El análisis documental, empleado para la revisión y extracción de datos por ejemplo libros de acta de calificaciones, Planeamiento de clases, lista de asistencias entre otros. Entrevista abierta a profundidad con alumnos y docentes de los primeros años de las carreras investigadas.

\section{Operacionalización de las variables}

\begin{tabular}{|c|c|c|}
\hline Variables & Conceptualización & Indicadores \\
\hline $\begin{array}{l}\text { Características socio } \\
\text { demográficas de los alumnos }\end{array}$ & $\begin{array}{l}\text { Contexto social y lugar de } \\
\text { procedencia del alumno }\end{array}$ & $\begin{array}{l}\text { Edad } \\
\text { Sexo } \\
\text { Lugar de procedencia } \\
\text { Lugar de Residencia }\end{array}$ \\
\hline Dificultades de adaptación & $\begin{array}{l}\text { Esta variable medirá las } \\
\text { capacidades de adaptación, del } \\
\text { alumno }\end{array}$ & $\begin{array}{l}\text { Vivienda } \\
\text { Horario de estudio } \\
\text { Equipos informáticos } \\
\text { Distancia para llegar a la } \\
\text { Universidad }\end{array}$ \\
\hline Rendimiento académico & $\begin{array}{l}\text { Consecuencias de la falta de } \\
\text { articulación }\end{array}$ & $\begin{array}{l}\text { Competencias } \\
\text { Habilidades necesarias para } \\
\text { incursionarse a la educación } \\
\text { superior. (...........) } \\
\text { Rendimiento escolar. } \\
\text { Excelente } \\
\text { Muy bueno } \\
\text { Bueno } \\
\text { Mala }\end{array}$ \\
\hline
\end{tabular}

\section{ELABORACIÓN DEL MARCO TEÓRICO}

\section{Antecedentes de la Investigación}

\subsection{Transición y articulación entre Educación Media y Superior}

Según Zamudio, González, y Velásquez (2016) en la investigación denominada. El dispositivo en la política educativa de la articulación de la Educación Media como la Superior. Caso IED María Mercedes Carranza. Universidad Pedagógica Nacional - UPN Bogotá, describen la política de articulación como un proceso que busca fortalecer tanto la educación media como la superior, propiciando las relaciones entre el sector productivo y educativo, y construir rutas formativas generando ganancias de tiempo y calidad para todos los actores, favoreciendo la continuidad de los jóvenes en el sector educativo a través de los ciclos propedéuticos y ampliando su posibilidad de insertarse laboralmente o generar un trabajo autónomo a través del emprendimiento. 
Avilés \& Tornel (2017) en su trabajo: Una experiencia piloto de articulación en ciencias básicas entre Educación Media Científico-Humanística y Educación Superior. Caso de la universidad del Bío-Bío, Chile, describe las estrategias de articulación curricular con la Enseñanza Media Científico -Humanista, se analizan los resultados obtenidos por el curso del Liceo Enrique Molina Garmendia que participó de manera piloto. Se concluye la necesidad de generar estrategias que permitan conocer deficiencias de contenidos de los estudiantes, con el propósito de elevar resultados académicos, fomentando su motivación y confianza en el proceso de preparación para el ciclo superior, así como también diseñar e implementar iniciativas que permitan conocer y dar tratamiento a factores intervinientes en la problemática de la deserción en la Educación Superior.

En la Universidad de Santiago se han generado instancias que han permitido democratizar el acceso efectivo a la educación superior, lo que implica un cambio de perfil del estudiante que ingresa a través de las distintas vías de acceso inclusivas, destacando la motivación y facilidad por el estudio. Sin embargo, las tasas de cobertura curricular de los establecimientos de los que provienen son bajas y, por lo tanto, tienen una mayor necesidad de nivelación (Miranda y Villarroel, 2015). Esto conlleva a enfrentar de manera distinta los procesos de transición y articulación de los estudiantes de la educación media a la superior, ya que la dificultad va más allá del acceso a los estudios superiores, centrándose en el ámbito de la comunicación- en la complejidad del desarrollo de habilidades y competencias lingüísticas que permitan a los estudiantes enfrentar con eficiencia las exigencias de las prácticas discursivas. Por lo tanto, la propuesta de articulación se focaliza en potenciar el desarrollo de habilidades comunicativas y en alfabetizar académica y disciplinariamente a los estudiantes, lo que implica una nueva forma de aprender, en contextos determinados y especializados (Gimeno y Segovia, 2016).

La educación superior es un bien de carácter estratégico para las naciones, en tanto es el vínculo indisoluble entre la generación de capital humano altamente capacitado y la producción y difusión de conocimientos que favorecen la conformación de sociedades más justas y economías más competitivas. Por ello, y sobre todo en estos tiempos en que el conocimiento es fundamental para el conjunto de actividades sociales, económicas y culturales, todos los países realizan importantes esfuerzos para contar con una educación superior que esté a la altura de las mejores prácticas y estándares internacionales en materia de formación profesional, investigación científica y desarrollo tecnológico (Fernández, 2017).

Según De Gatica, Romero, Bort, \& de Gatica (2019) afirman que la transición entre niveles educativos es una instancia crítica que precisa ser abordada desde distintas aristas. 
Puntualmente, el pasaje entre el nivel secundario y el nivel superior suele ser particularmente problemático y requiere de la vinculación entre las distintas instituciones involucradas.

En este contexto, la articulación con otros niveles del sistema educativo es parte de una de las problemáticas que requieren la búsqueda de posibilidades y estrategias que permitan resolver la segmentación para favorecer a personas de las franjas socioeconómicas más vulnerables y dar espacio para que todos los estudiantes realicen una trayectoria académica que responda a sus expectativas y les brinde la posibilidad de alcanzar el ascenso social.

Entonces, es claro que favorecer el acceso a la universidad, a partir una política de educación pensada desde la inclusión social, implica el desarrollo de estrategias para atenuar el abandono de la escuela secundaria y así lograr el pasaje efectivo entre niveles permitiendo a los jóvenes continuar sus estudios (De Gatica, Romero, Bort, \& de Gatica, 2019).

\subsection{Articulación}

Al ubicar dentro del contexto educativo el concepto de la articulación media con la superior, según el documento elaborado por el Ministerio de Educación Nacional y el Servicio Nacional de Aprendizaje SENA (2012, como se citó en Rubio, Mejía, \& Huertas, 2015). afirman que:

La articulación es un proceso pedagógico y de gestión concertado que favorece el acceso, permanencia y movilidad de los estudiantes entre la educación media y otros niveles y ofertas educativas; este proceso genera el reconocimiento de los aprendizajes obtenidos en distintos escenarios formativos y el mejoramiento continuo de la pertinencia y calidad de la educación media, la educación superior, la formación profesional integral y la educación para el trabajo y el desarrollo humano. (p.7)

Otros autores como Olga Beatriz Cordero de Barrientos (2003, como se citó en Rubio, Mejía, \& Huertas, (2015).

La articulación educativa es un modelo teórico-práctico de organización y gestión, aplicable a los distintos niveles y campos del sistema educativo, dinámico, flexible, abierto y complejo; que se construye y sostiene en procesos de interacción sistémica, entre todos los actores educativos, para establecer relaciones funcionales y significativas entre los componentes del campo de la educación, curriculares y metas curriculares, a fin de lograr metas consensuadas de la enseñanza (p.745).

De acuerdo con lo señalado por Gaete y Morales (2011, como se citó en Gallegos \& Rodríguez 2017). Estos dos niveles formativos, Educación Media y Educación Superior, deberían lograr una mayor flexibilidad en el currículum para permitir así la posibilidad de una articulación. En 
este sentido, se entiende como tal al "proceso de unión, enlace, continuidad entre los distintos niveles del sistema educativo".

Ander-Egg, (1997 como se citó en Gallegos \& Rodríguez 2017). El proceso de articulación debe considerar las acciones necesarias, entre las Instituciones participantes, que les permita a los estudiantes pasar con mayor facilidad de un nivel a otro en su proceso formativo. En este contexto, se hace necesario institucionalizar el proceso de articulación para contar con procedimientos y responsables claramente declarados en cada una de las organizaciones y así facilitar su proceso.

Ahora bien, existen dos tipos de articulación: Una articulación horizontal y una articulación vertical. De acuerdo con el Centro de Documentación e Información Educativa del Ministerio de Educación de Tucumán (2008 como se citó en Gallegos \& Rodríguez 2017) la articulación horizontal es aquella en la cual los conceptos, procedimientos y valores se conectan entre sí para garantizar la coherencia al interior de cada campo o áreas curriculares, y en la totalidad de los campos o áreas curriculares. Por su parte, la articulación vertical es aquella que busca facilitar y garantizar propuestas curriculares jurisdiccionales e institucionales que tengan en cuenta la lógica interna de las distintas disciplinas y áreas del conocimiento, la evolución personal de los y las estudiantes, así como la movilidad de la población escolar.

Gaete y Morales (2011 como se citó en Gallegos \& Rodríguez 2017) definen como articulación horizontal la organización del currículo de un mismo nivel formativo, de tal manera que un plan de estudio no sea exclusivo de una carrera ni tenga las mismas y únicas vías de entrada y salida, sino que pueda ser cursado por estudiantes de diversas carreras y con ello facilitar el cambio de una carrera a otra, así como la doble titulación. Mientras que la articulación vertical se refiere a las posibilidades de transitar fluidamente de un nivel a otro dentro del sistema educativo y con reconocimiento del $100 \%$ de los estudios previos, esto debido a que los programas formativos se encuentran armonizados curricularmente.

\subsection{Dificultades para la institución educativa la articulación}

Según Restrepo y García (2017) con este escenario de fondo se detalla que la dificultad para las instituciones educativas que disponen de estos programas no radica necesariamente en la falta de conceptualización, sino en la pertinencia de dichos programas en función de dos escenarios: 1), la articulación con los procesos y programas de las instituciones de educación superior; y 2), la absorción de los estudiantes formados en el mercado productivo del país (Restrepo y García, 2017). 


\subsection{Raíces del Conservadurismo y la articulación de la Educción Media y la Universitaria}

La reforma educativa no fue resultado de un proyecto gestado por las fuerzas políticas que asumieron el control del poder en 1989. Extrañamente, fue una respuesta de las nuevas autoridades a un vigoroso reclamo de la población que vio en la educación el principal instrumento para organizar y sustentar una profunda transformación social y política. Una expresión de este vigoroso ensamble fue la decisión del primer gobierno de la transición de integrar el Consejo Asesor de la Reforma Educativa que tendría un papel crucial en la gestación y desenvolvimiento de la reforma de la educación paraguaya - con renombradas figuras nacionales pertenecientes a diversas corrientes de pensamiento y campos de actividad y que, además, tuvieron una destacada militancia en la defensa de los principios democráticos.

¿Cómo se explica que la universidad paraguaya estuviera excluida de esta trascendental movilización ciudadana, que buscaba la transformación de la educación con miras a una radical transformación de la sociedad? La universidad paraguaya se mantuvo distanciada e indiferente al vasto esfuerzo de cambio llevado a cabo en el nivel básico y medio; y aún hoy día su contribución para encarar y orientar institucionalmente a enfrentar los grandes desafíos nacionales, sigue siendo extremadamente limitada. Más aún, la resistencia a emprender cambios estructurales, sea en el plano del sistema de gobierno o de la estructura y orientación académica, en general, reduce su avance a impulsos meramente inerciales. ¿Cuáles son las raíces de la preeminencia que sigue teniendo en la universidad paraguaya esta postura conservadora? Posiblemente, la inercia prevaleciente responde a una multiplicidad de factores cuya exposición reclamaría un análisis más detenido. Al respecto, en el contexto de estas reflexiones resulta pertinente mencionar dos fenómenos estrechamente ligados a la práctica conservadora subyacente en la universidad paraguaya: una, el papel real desempeñado por el principio de autonomía universitaria y otra, la mediatización (Bordas 2004, como se citó en Rivarola 2008).

\subsubsection{Una antigua disensión, la articulación}

A lo largo de su historia, el sistema educativo paraguayo permaneció prácticamente escindido en dos estamentos: uno, que cubría la educación escolar básica y la media; y otro, la educación superior universitaria. La responsabilidad de organización, orientación y funcionamiento de la primera fue y sigue siendo una competencia del Ministerio de Educación y Cultura, en tanto que la que corresponde al estamento universitario sigue manejándose por la ley de universidades que le atribuye una condición de autonomía destinada a establecer y regular su propio gobierno. 
El marco jurídico institucional tuvo su origen en la primera ley de universidades que se sancionó el 29 de junio de 1929 (Ley No. 1046). (Cubilla, 2000, como se citó en Rivarola 2008). La misma, inspirada fuertemente por la reforma de Córdoba, estableció el principio de autonomía, la que fue reiterándose en la totalidad de las leyes promulgadas relativas a este estamento del sistema educativo nacional. Vista desde otra perspectiva, la baja cobertura que ha sido, hasta época muy reciente, una de las características más resaltantes de la educación superior, ensanchó aún más la distancia entre uno y otro sector educativo. Recién con la actual reforma educativa al iniciarse la década del noventa, el Ministerio de Educación comienza a involucrarse de manera directa con el sistema universitario, en particular a través de la responsabilidad que le correspondió por mandato del Mercosur Educativo, en la puesta en marcha de la Agencia Nacional de Evaluación y Acreditación. Posteriormente, el gobierno nacional da un paso importante al constituir por ley nacional el Consejo Nacional de Reforma de la Educación Superior, de carácter interinstitucional, integrada por el Ministerio de Educación y Cultura (MEC); el Consejo Nacional de Educación y Cultura (CONEC); y el Consejo de Rectores (Cubilla, 2006, como se citó en Rivarola 2008). La Educación Superior se planea una visión social con demarcaciones axiológica, que enfatiza revelación de la pertinencia en términos de la transición hacia sociedades del conocimiento. Su construcción lógica se basa en formar un ciudadano trasformador de la realidad como tendencia del siglo XXI (CRES, 2018).

A pesar de estas determinaciones que apuntaban a lograr un avance en la articulación entre el sistema universitario y el correspondiente a la enseñanza básica y media, apenas iniciada la reforma educativa, se ahondó la fisura entre ambos niveles educativos, al declinar el estamento universitario a participar en el proceso de reforma que se impulsa desde el Ministerio de Educación y Cultura. La dirigencia universitaria, invocando el principio de autonomía desistió, de cualquier intento de involucrarse con la reforma educativa (Rivarola 2008).

\subsection{Plan Estratégico de la Reforma Educativa, Paraguay 2020}

El presente plan tuvo su inicio en el año 1996, en ese entonces por el todavía Ministerio de Educación y Culto. El plan tuvo por objetivo presentar la visión del sistema educativo que se quiere.

El propósito de este plan fue lograr la asimilación de valores y desarrollar las competencias fundamentales para una vida digna, en todos los hombres y mujeres en el país.

Los resultados esperados de este Plan fueron: 1- Mejorar la calidad y pertinencia de la Educación Básica (Escolar Básica y General Básica); 2- Mejorar la calidad y pertinencia de la 
Educación media y técnica; 3- Mejorar la calidad y pertinencia de la formación profesional, y;4 Mejorar la calidad y pertinencia de la Educación Superior. Estaba dividido en dos áreas prioritarias, Educación Escolar Básica y Educación General Básica (Sterling, 2019).

\subsubsection{Plan Estratégico de Educación, Paraguay 2020}

Revisión Nacional 2015 de la Educación para Todos: Paraguay." Actualizado (2008-2020) En el 2008, se actualiza el Plan 2020 como respuestas a las transformaciones de la sociedad y a los avances del conocimiento, que tuvo por objetivo general garantizar el acceso, el mejoramiento de la calidad, la eficiencia y la equidad de la educación paraguaya.

\subsubsection{Plan Nacional de Educación 2024 "Hacia el centenario de la Escuela Nueva de Ramón Indalecio Cardozo"}

Este marco constituye un replanteamiento de la política y gestión de educación que el MEC realiza, se involucra a todos los niveles del sistema educativo, desde la educación básica a la educación superior, incluyendo a las diferentes instancias de gestión administrativa a escala central y departamental (Sterling, 2019).

Con estos datos se pueden evidenciar las características sociales del estudiante en cuanto a la cobertura educativa, a quiénes no se hace fácil sostenerse en la ciudad y cubrir sus gastos universitarios.

Al respecto la UNESCO, (1998). Establece que el acceso a la educación es un derecho fundamental que está sustentado en los principios de obligatoriedad y gratuidad, y en el derecho a la no discriminación. Asimismo, la educación es un medio potente y dinámico, que faculta a las personas que se encuentran marginadas socialmente, a salir de las carencias económicas y culturales en que se encuentran, por su propio esfuerzo -siempre y cuando se otorguen las condiciones apropiadas.

Muchos de los estudiantes tienen dificultades en el aprendizaje, respecto a las causas de estas dificultades, se citan, la falla en la comunicación docente-estudiantes, falta de dedicación del estudiante, la falta de articulación entre lo académico y lo administrativo (establecen horario para las últimas horas de asignaturas que requieren mucha atención y donde los alumnos ya se encuentran cansados al igual que el docente).

Los beneficios de la articulación en el desarrollo de las capacidades en forma sistemática, contribuyendo al desarrollo de las capacidades desarrolladas todas las áreas del saber, sin embargo, existe la necesidad de mayor énfasis a este delicado proceso para evitar el quiebre en el proceso de pase de la Educación Media y la Universitaria. 
Según el Programa País-OEI Py (2019-2020). La calidad de la educación superior sigue siendo un área de trabajo a seguir mejorando; es preciso mejorar la coordinación en la planificación ente la EEB y EM y la EU ya que no existe claridad y consistencia con relación a las formas de integrar, articular y armonizar los programas educativos; es preciso mejorar la relevancia y el contenido de los currículos escolares de formación profesional ya que están desconectados con las necesidades del mercado laboral. No obstante, la expansión de la Educación Superior en Paraguay durante la última década ha sido elevada.

La adaptación al medio del nuevo estudiante universitario será más llevadera con apoyo de docentes y autoridades académicas, quizás con actitudes más humanas y tratos más agradable, los estudiantes estarán abiertos al aprendizaje y si esto no fuese posible, pondrían más de sí para llegar a los objetivos propuestos por el docente, considerando que ni la situación social facilita el aprendizaje de los alumnos, por lo tanto, su adaptación al medio tampoco será muy fácil.

Existen situaciones de estudiantes que tienen dificultades para adaptarte al nuevo ambiente, otro factor es la movilidad que tienen en cuanto a transporte y distancia, considerando que la distancia desde sus resinifica a la facultad no siempre es buena o que muchos tienen que llegar a la facultad caminando. Así también un punto muy importante es la capacidad que tiene el estudiante para establecer nuevas amistades.

Según Soares, Almeida y Guisande, (2011). En las últimas décadas, las cuestiones de la transición y la adaptación de los jóvenes a las presiones y a los desafíos de la vida universitaria han sido objeto de gran interés en la literatura internacional (Astin, 1993; Baker y Siryk, 1984; Chickering y Reisser, 1993; Pascarella y Terenzini, 2005; Tinto, 1993)

\section{HALLAZGOS Y DISCUSIÓN}

A continuación, se presenta los resultados conforme a las variables estudiadas.

\section{Variable: Característica sociodemográfica de los alumnos de la zona periférica}

- ¿Quién te ayuda a solventar sus gastos? 


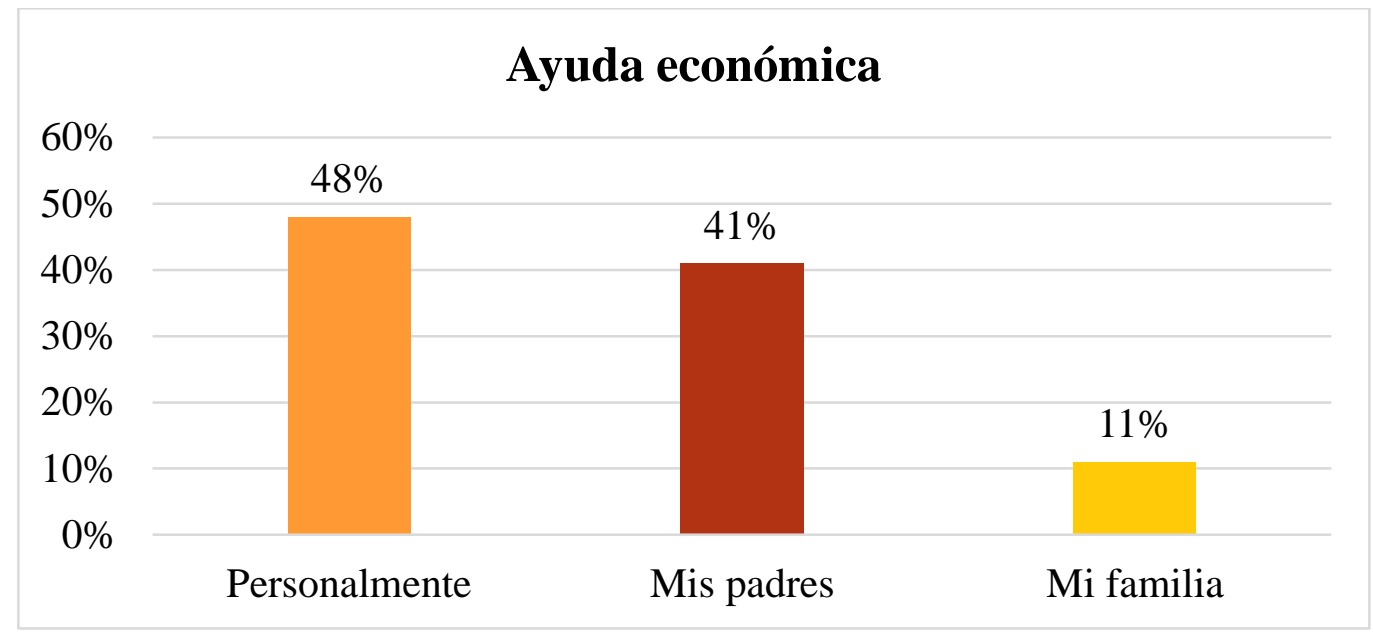

- ¿Alcanzas a cubrir los gastos mínimos de la facultad?

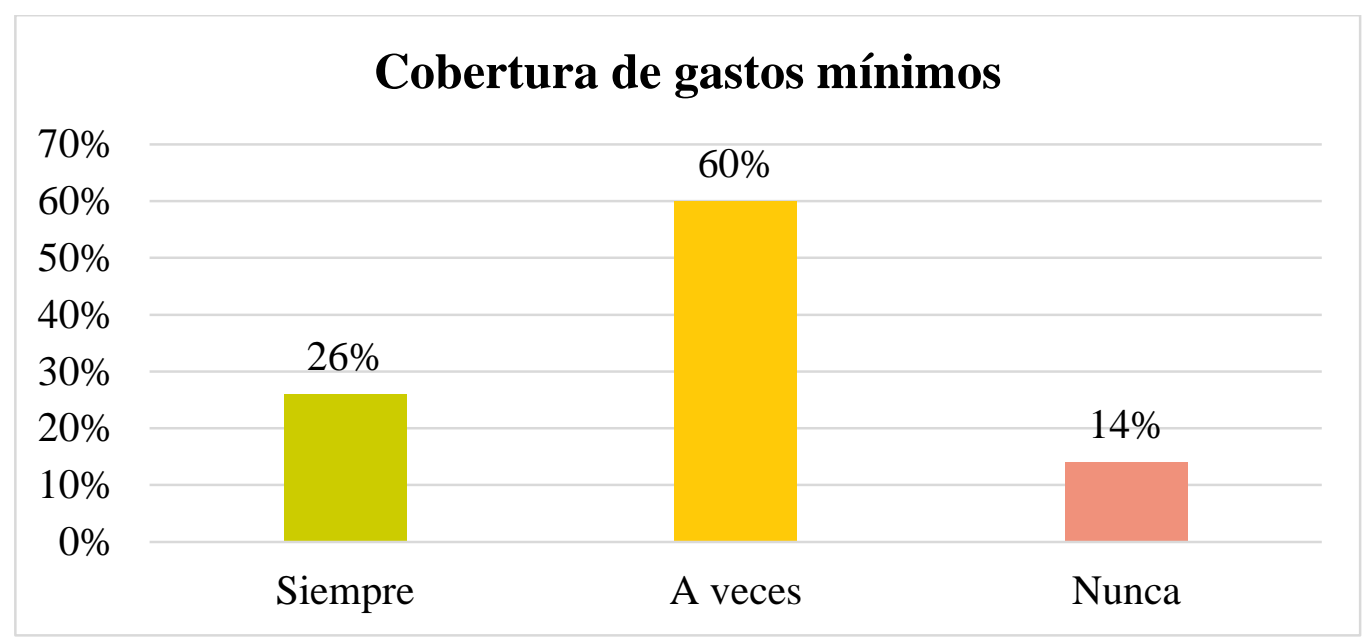

Se puede observar en el presente gráfico que por lo general son los mismos alumnos quienes solventa sus gastos, $48 \%$; a otros les ayuda sus padres, $41 \%$; como también existen quienes reciben ayuda de algún familiar para solventar sus gastos universitarios.

En cuanto a la cobertura de gastos mínimos, solo a veces alcanzar subirlas, $60 \%$, otros dicen siempre, $26 \%$ y el $14 \%$ nunca.

Con estos datos se pueden evidencia las características sociales del estudiante en cuanto a la cobertura educativa, a quienes no se hace fácil sostenerse en la ciudad y cubrir sus gastos universitarios.

Al respecto la UNESCO, (1998). Establece que el acceso a la educación es un derecho fundamental que está sustentado en los principios de obligatoriedad y gratuidad, y en el derecho a la no discriminación. Asimismo, la educación es un medio potente y dinámico, que faculta a las personas que se encuentran marginadas socialmente, a salir de las carencias económicas y culturales en que se encuentran, por su propio esfuerzo -siempre y cuando se otorguen las condiciones apropiadas. 
Variable: Obstáculos que enfrentan los estudiantes durante los procesos de interacción sistémica,

- ¿Tienes dificultades en el aprendizaje de las clases?

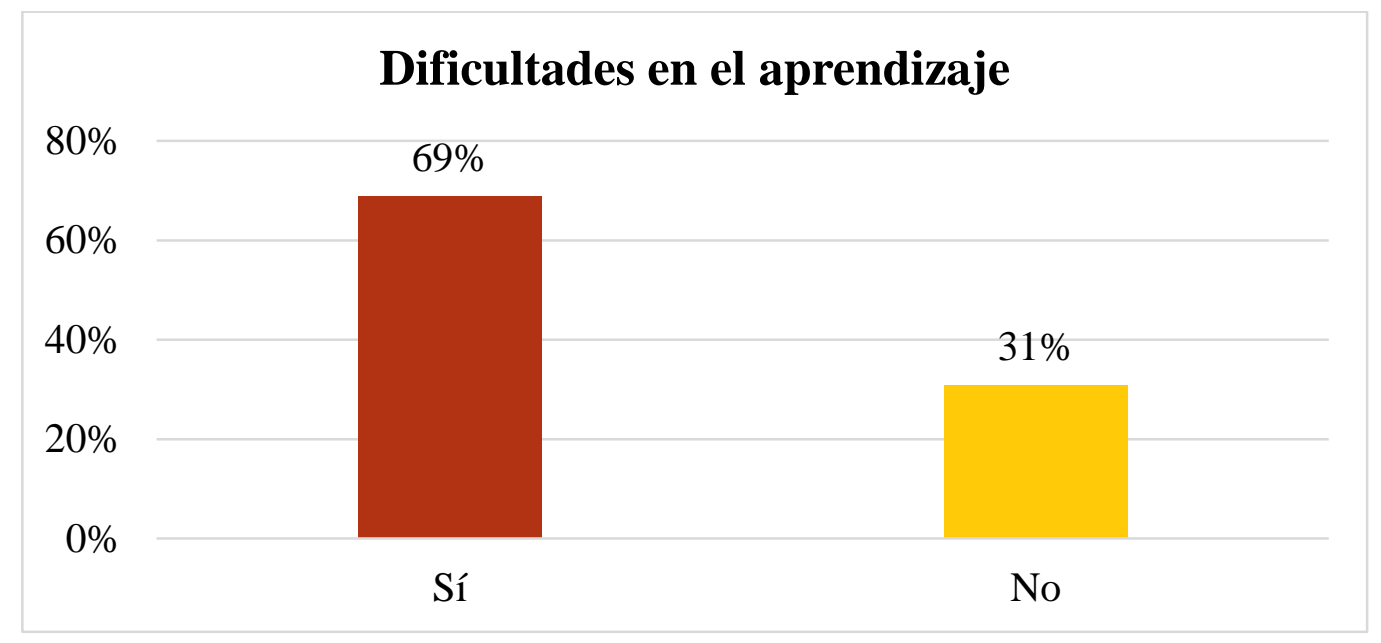

- Las dificultades que tienes son debido a:

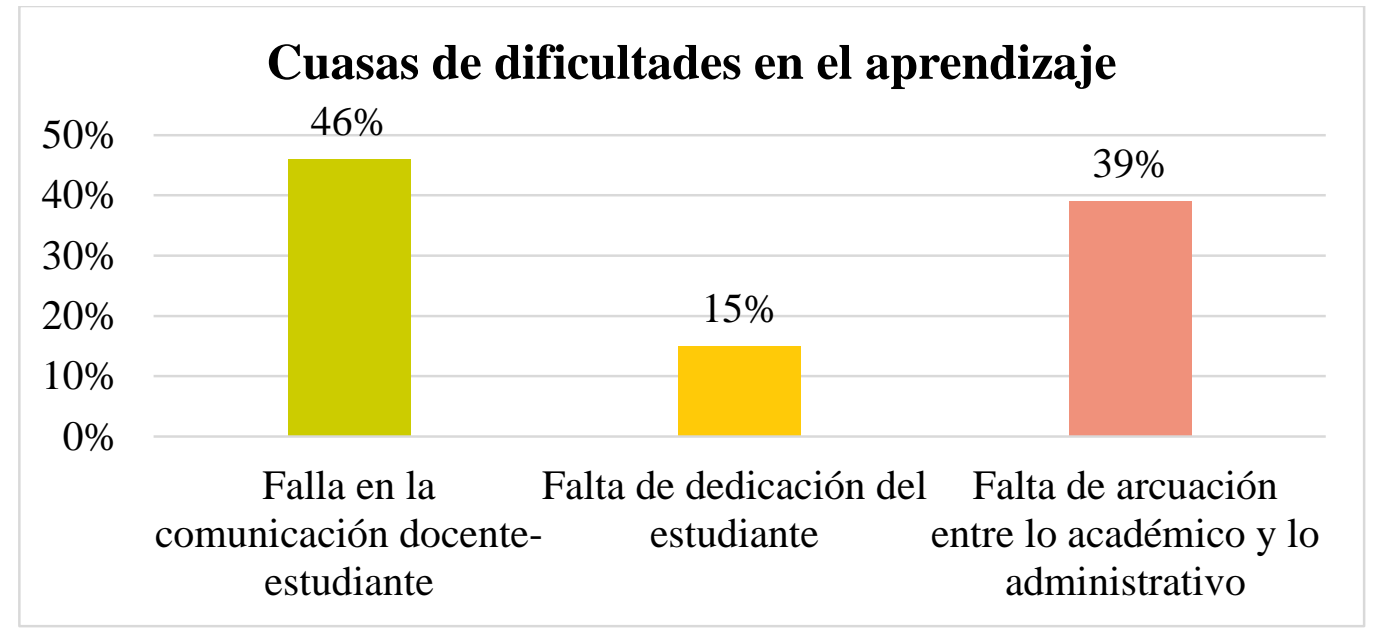

Muchos de los estudiantes tienen dificultades en el aprendizaje 69\%, y el 31\% dicen que no encuentran dificultad en el aprendizaje. Respeto a las causas de estas dificultades sobre sale la falla en la comunicación docente-estudiantes $46 \%$, falta de dedicación del estudiante $15 \%$ y el $39 \%$ es la falta de articulación entre lo académico y lo administrativo (establecen horario para las últimas horas de asignaturas que requieren mucha atención y donde los alumnos ya se encuentran cansados al igual que el docente).

Los beneficios de la articulación en el desarrollo de las capacidades en forma sistemática, contribuyendo al desarrollo de las capacidades desarrolladas todas las áreas del saber, sin embargo, existe la necesidad de mayor énfasis a este delicado proceso para evitar el quiebre en el proceso de pase de la Educación Media y la Universitaria. 
Según el Programa País-OEI Py (2019-2020). La calidad de la educación superior sigue siendo un área de trabajo a seguir mejorando; es preciso mejorar la coordinación en la planificación ente la EEB y EM y la FU ya que no existe claridad y consistencia con relación a las formas de integrar, articular y armonizar los programas educativos; es preciso mejorar la relevancia y el contenido de los currículos escolares de formación profesional ya que están desconectados con las necesidades del mercado laboral. No obstante, la expansión de la Educación Superior en Paraguay durante la última década ha sido elevada.

Variable: Problemas de adaptación de los alumnos al nuevo ambiente

- ¿Tiene dificultades para adaptarte al nuevo ambiente?

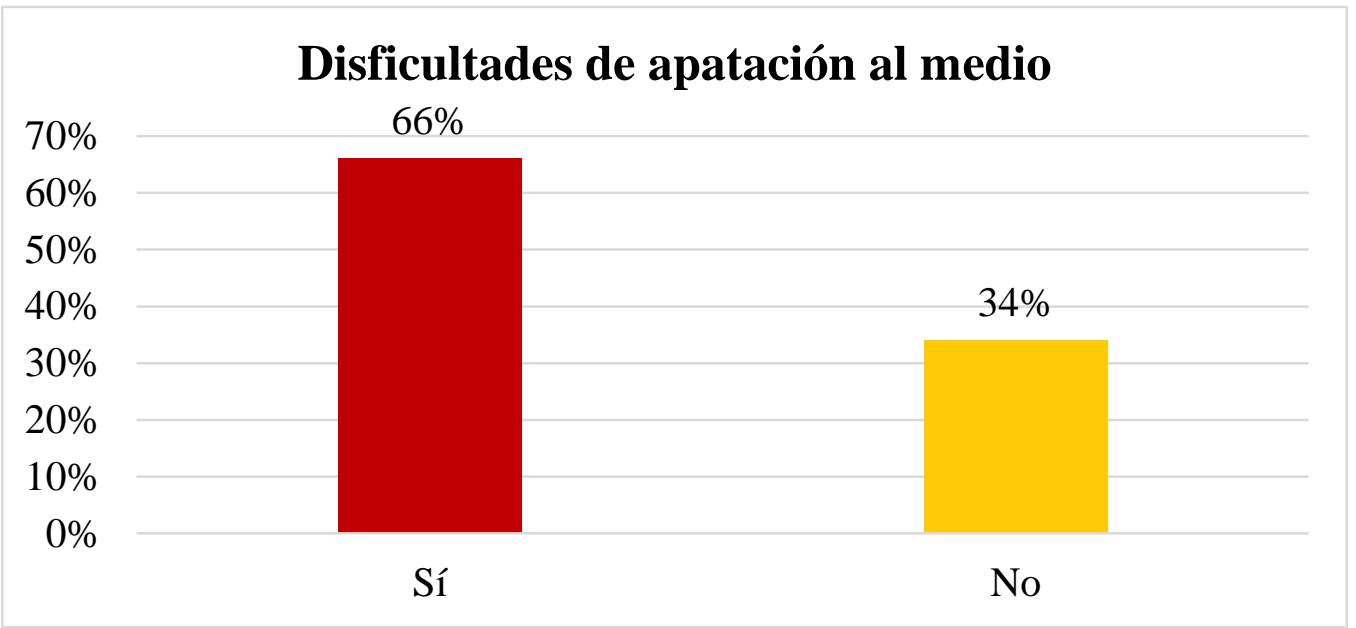

- ¿Como calificarías la movilidad que tienen en cuanto a trasporte y distancia?

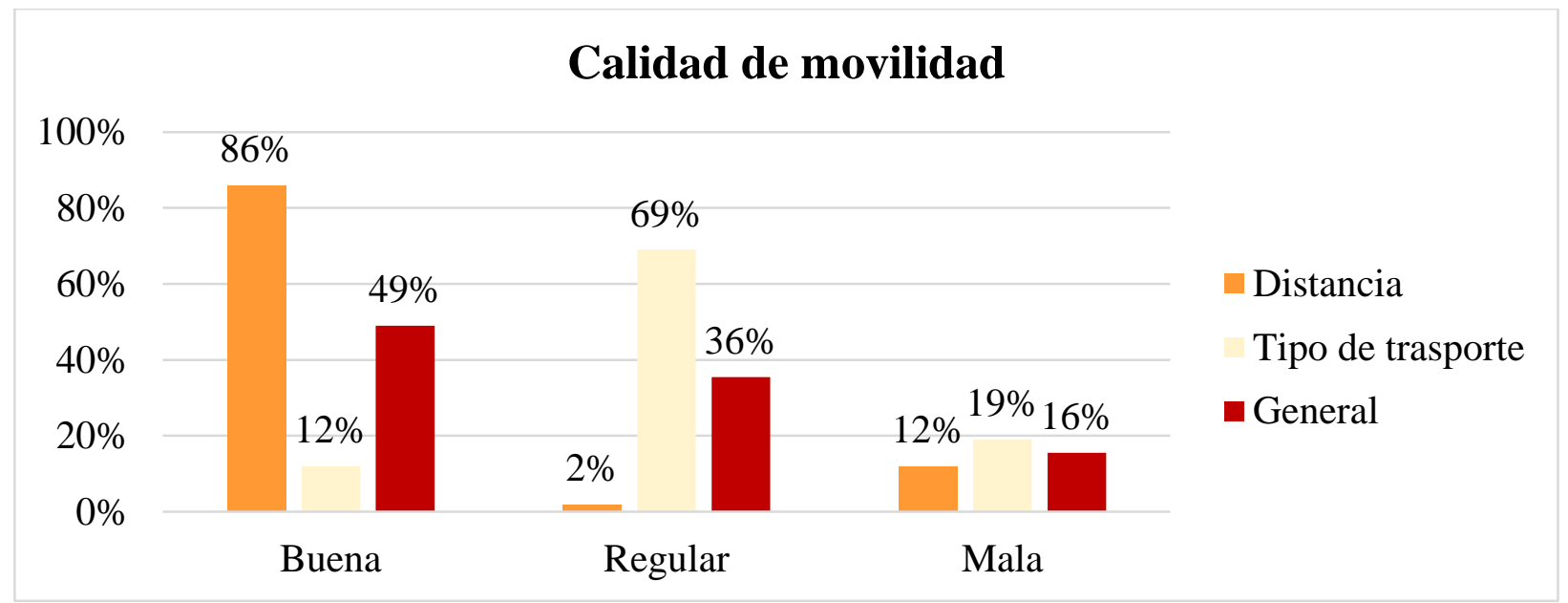

- ¿Has podido establecer nuevas amistades? 


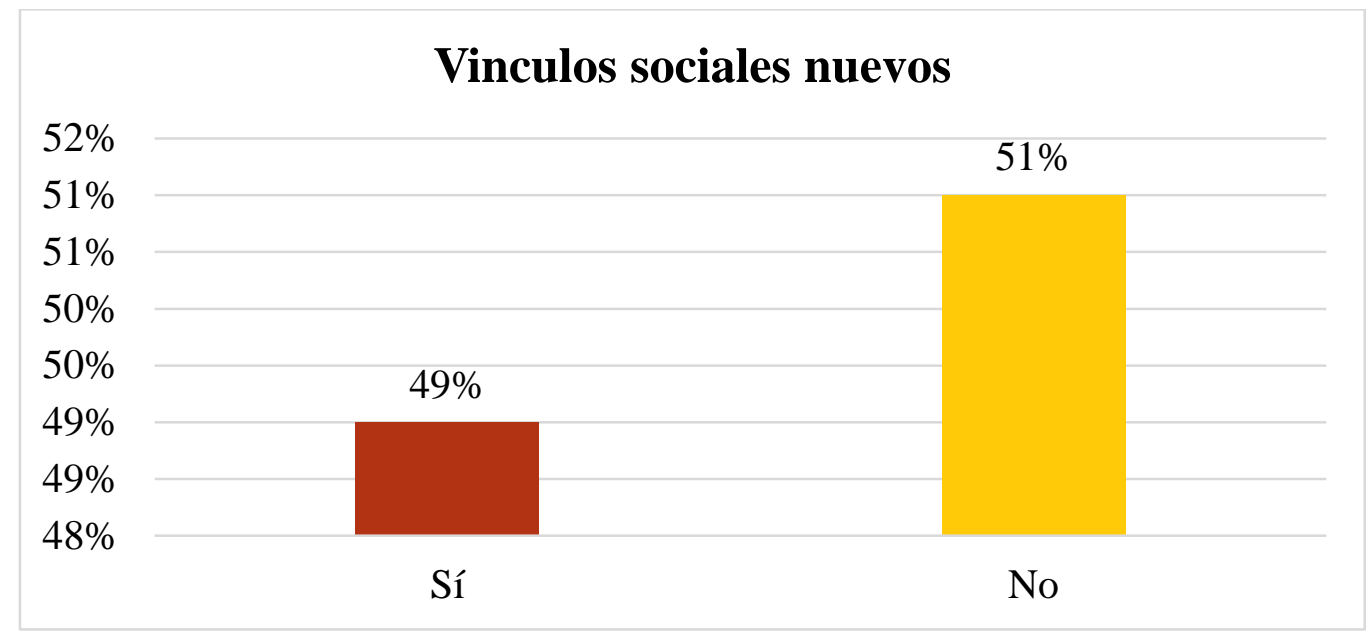

Se puede observar en el presente gráfico que el 69\% de los estudiantes sí tiene dificultades para adaptarte al nuevo ambiente y el $34 \%$ dicen que no.

Respecto a la movilidad que tienen en cuanto a trasporte y distancia, el $89 \%$ consideran que la distancia desde sus resinifica a la facultad es buena, el tipo de trasporté solo 69\% dicen regular siendo que muchos tienen que llegar a la facultad caminado.

No todos los estudiantes han podido establecer nuevas amistades, $51 \%$ y soplo el $49 \%$ dicen que esto fue posible.

La adaptación al medio del nuevo estudiante universitario será mas llevadera con apoyo de docentes y autoridades académicas, quizás con actitudes más humanas y tratos más agradable, los estudiantes estarán abiertos al aprendizaje y si esto no fuese posible, pondrían más de si para llegar a los objeticos propuestos por el docente, considerando que ni la situación social facilita el aprendizaje de los alumnos, por lo tanto, su adaptación al medio tampoco será muy fácil.

Según Soares, Almeida y Guisande, (2011). En las últimas décadas, las cuestiones de la transición y la adaptación de los jóvenes a las presiones y a los desafíos de la vida universitaria han sido objeto de gran interés en la literatura internacional (Astin, 1993; Baker y Siryk, 1984; Chickering y Reisser, 1993; Pascarella y Terenzini, 2005; Tinto, 1993).

\section{CONCLUSIÓN}

La falta de articulación entre el Nivel Medio y la universitaria es una realidad que se vive en las aulas, y que sin embargo no se la dimensiona en su real magnitud, pues de lo contrario dejaría de ser considerado un hecho que habitualmente es tratado con medidas improvisadas, casuales, que no pasan de ser meros comentarios entre directivos y docentes a un tema serio de interés, objeto de propuestas bien planificadas, coordinadas y ejecutadas desde la administración central pero con la participación real y comprometida de los principales responsables del hecho educativo. 
La falta de articulación en los primeros años de la educación universitaria y la educación media es una realidad evidenciada sobre todo para una población con menos posibilidades educativas como son las zonas periféricas, por lo tanto, la articulación ocupa de una problemática actual, emergente y preocupante, considerando que la propuesta educativa proveniente de la administración central, es decir, del Ministerio de Educación y Cultura, está diseñada como para evitar el quiebre del proceso en el paso del estudiante de un nivel a otro. Sin embargo, al mencionarse la falta de articulación implícitamente se reconoce la falla en el diseño de la propuesta educativa del nivel medio al nivel universitario.

La Constitución Nacional en su Art. 73 "Del derecho a la educación y de sus fines”, establece que "Toda persona tiene derecho a la educación integral y permanente que como sistema y proceso se realiza en el contexto de la cultura de la comunidad". En concordancia con este postulado constitucional y de acuerdo con los principios de la Educación que propugna una “educación para todos" que satisfaga las necesidades básicas de cada persona, nuestro país pretende brindar una oferta educativa que atienda de manera integral y permanente a los alumnos y alumnas.

\section{REFERENCIAS BIBLIOGRAFICAS}

"Revisión Nacional 2015 de la Educación para Todos: Paraguay." Para obtener más información, contactar con: efa2015reviews@unesco.org

Arias, F. (2006). “El Proyecto de Investigación” 6 Edición. Editorial: Episteme. Venezuela Avilés Gavilán, Grecia, \& Tornel Cifuentes, Mabel (2017). Una experiencia piloto de articulación en ciencias básicas entre educación media científico-humanística y educación superior. Resultados del caso de la universidad del Bío-Bío, Chile. Espacios en Blanco. Revista de Educación, (27),129-151.[fecha de Consulta 20 de Diciembre de 2019]. ISSN: 1515-9485. Disponible en: https://www.redalyc.org/articulo.oa?id=3845/384551991006

CRES (2018). Colección CRES 2018 - Tendencias de la Educación Superior en América Latina y el Caribe 2018. Recuperado de http://www.iesalc.unesco.org/2019/07/17/coleccion-cres-2018-tendencias-de-laeducacion-superior-en-america-latina-y-el-caribe-2018/

De Gatica, A, Romero, M M, Bort, L, \& de Gatica, N. (2019). Representaciones contrapuestas: la escuela secundaria piensa a la universidad. InterCambios. Dilemas y 
transiciones de la Educación Superior, 6(2), 18-27. Epub 01 de diciembre de 2019.https://dx.doi.org/10.29156/inter.6.2.3

Fernández Fassnacht, E (2017). Una mirada a los desafíos de la educación superior en México. Innovación educativa (México, DF), 17(74), 183-207. Recuperado en 23 de enero de 2020, de http://www.scielo.org.mx/scielo.php?script=sci_arttext\&pid=S1665$26732017000200183 \& \operatorname{lng}=\mathrm{es} \& \ln \mathrm{ln}=\mathrm{es}$.

Figueroa, L, Maillard, B, Veliz, N, Toledo, S, González, M, (2016). V CLABES Quinta Conferencia Latinoamericana Sobre el Abandono en la Educación Superior la Experiencia de los Programas Propedéuticos y su articulación con la Escuela Línea Temática: Articulación de la educación superior con las enseñanzas medias. Tipo de comunicación: experiencia.

Gallegos Muñoz, C, \& Rodríguez Quezada, E. (2017). Formación contable en Chile "una propuesta de articulación curricular”. Actualidades Investigativas en Educación, 17(3), 532-564. https://dx.doi.org/10.15517/aie.v17i3.29988

Gimeno, M y Segovia, K. (2016). Programa de Acceso Inclusivo, Equidad y Permanencia Universidad de Santiago de Chile - CHILE. Articulación entre Educación Media y Superior Bajo la Metodología de Aprendizaje y Servicio: Experiencia de la Academia Pace Usach Línea Temática: Articulación de la educación superior con las enseñanzas medias.

Hernández, Dr. Fernández, Dra. Baptista. “Metodología de la Investigación”. McGraw Hill 1994. Colombia.

Hernández, Dr. Fernández, Dra. Baptista. “Metodología de la Investigación” Cuarta Edición McGraw Hill 2006. México.

Lleixà, T.; Gros, B.; Mauri, T.; Medina, J.L (eds.) (2018) Educación 2018-2020. Retos, tendencias y compromisos. Barcelona: IRE-UB Descargable desde:

http://www.ub.edu/ire/ ISBN: 978-84-09-02933-4

Programa País-OEI Paraguay (2019-2020). Organización de Estados Iberoamericanos para la Educación, la Ciencia y la Cultura Oficina Regional en Paraguay. Recuperado de https://oei.org.py/uploads/files/news/OEI-Institutional/615/programa-pais-oei-paraguay2019-2020a.pdf

Restrepo Medina, L, \& García Morales, D (2017). Evaluación de los programas técnicos y de profundización de la educación media en la Institución Educativa Javiera Londoño (sede Medellín ). Revista Virtual Universidad Católica del Norte, (51),64-75.[fecha de 
Consulta 20 de Diciembre de 2019]. ISSN: 0124-5821. Disponible

en: https://www.redalyc.org/articulo.oa?id=1942/194252398005.

Rivarola, Domingo. (2008). La universidad paraguaya, hoy. Avaliação: Revista da Avaliação da Educação Superior (Campinas), 13(2), 533-578. https://dx.doi.org/10.1590/S141440772008000200014

Rubio, R. G., Mejía, H. E. \& Huertas, V. M. (2015). Articulación de la educación media con la educación superior: instrumento administrativo para el sector educativo de la ciudad de Ibagué. Praxis. Vol. 11, 76 - 88

Sampieri, H. (2003). “Metodología de la Investigación”. Editorial: McGraw-Hill. México

Soares, A.P Almeida, L.; Guisande, M. (2011) Ambiente Académico y Adaptación a la Universidad: Un Estudio con estudiantes de $1^{\circ}$ año de la Universidad do Minho. Revista Iberoamericana de Psicología y Salud, vol. 2, núm. 1, enero, 2011, pp. 99-121 Sociedad Universitaria de Investigación en Psicología y Salud A Coruña, España.

Sterling Collazos, J.M (2019). Articulación entre la Educación Superior y la Educación

Rural. Estudio de caso en Zipaquirá, Colombia. Conrado, 15(68), 263-266. Epub 02 de septiembre de 2019. Recuperado en 26 de enero de 2020, de http://scielo.sld.cu/scielo.php?script=sci_arttext\&pid=S1990$\underline{86442019000300263 \& \operatorname{lng}=\mathrm{es} \& \operatorname{tlng}=\mathrm{es} .}$

Tamayo y Tamayo M. (1998) “Metodología de la Investigación” Cuarta Edición McGraw Hill México.

Zamudio Clavijo, H, González, J. y Velásquez M (2016). El dispositivo en la política educativa de la articulación de la media con el superior estudio de caso IED María Mercedes Carranza. Trabajo de Investigación Maestría en Desarrollo Educativo y Social. Convenio Fundación Centro internacional de Educación y Desarrollo Humano Cinde - Universidad Pedagógica Nacional - UPN Bogotá D.C. 